\title{
Impact of Awareness to Control Malaria Disease: A Mathematical Modeling Approach
}

\author{
Malik Muhammad Ibrahim (D), ${ }^{1}$ Muhammad Ahmad Kamran (D), \\ Malik Muhammad Naeem Mannan $\mathbb{D}^{3},{ }^{3}$ Sangil Kim $\mathbb{D D}^{1}$ and Il Hyo Jung $\mathbb{D}^{1,4}$ \\ ${ }^{1}$ Department of Mathematics, Pusan National University, Busan 46241, Republic of Korea \\ ${ }^{2}$ Department of Cogno-Mechatronics, Pusan National University, Busan 46241, Republic of Korea \\ ${ }^{3}$ School of Allied Health Sciences, Griffith University, Gold Coast, Australia \\ ${ }^{4}$ Finance Fishery Manufacture Industrial Mathematics Center on Big Data, Pusan National University, \\ Busan 46241, Republic of Korea \\ Correspondence should be addressed to Sangil Kim; sangil.kim@pusan.ac.kr and Il Hyo Jung; ilhjung@pusan.ac.kr
}

Received 29 April 2020; Revised 30 July 2020; Accepted 10 August 2020; Published 28 October 2020

Academic Editor: Tongqian Zhang

Copyright (c) 2020 Malik Muhammad Ibrahim et al. This is an open access article distributed under the Creative Commons Attribution License, which permits unrestricted use, distribution, and reproduction in any medium, provided the original work is properly cited.

\begin{abstract}
The mathematical modeling of malaria disease has a crucial role in understanding the insights of the transmission dynamics and corresponding appropriate prevention strategies. In this study, a novel nonlinear mathematical model for malaria disease has been proposed. To prevent the disease, we divided the infected population into two groups, unaware and aware infected individuals. The growth rate of awareness programs impacting the population is assumed to be proportional to the unaware infected individuals. It is further assumed that, due to the effect of awareness campaign, the aware infected individuals avoid contact with mosquitoes. The positivity and the boundedness of solutions have been derived through the completing differential process. Local and global stability analysis of disease-free equilibrium has been investigated via basic reproductive number $R_{0}$, if $R_{0}<1$, the system is stable otherwise unstable. The existence of the unique endemic equilibrium has been also determined under certain conditions. The solution to the proposed model is derived through an iterative numerical technique, the Runge-Kutta method. The proposed model is simulated for different numeric values of the population of humans and anopheles in each class. The results show that a significant increase in the population of susceptible humans is achieved in addition to the decrease in the population of the infected mosquitoes.
\end{abstract}

\section{Introduction}

Malaria is an ancient disease with challenging health issues. The tropical regions such as Africa, Asia, and America are favorable for the rapid spread of this disease [1]. In 2018, there are estimated two-hundred and twenty-eight million cases of malaria around the world. This deadly disease is the root cause of the death of four-hundred-five thousand people according to the World Health Organization (WHO) 2019 world malaria report [2]. This disease is originated by the plasmodium parasite. The transmission of this infection to human body is by the bite of a female mosquito. Medical symptoms such as a rise in the body temperature, fatigue, pain, shivering, and sweats may occur within a few days after an infected mosquito bite. Till the time, there is no effective vaccine developed and some existing antimalarial drugs are losing their effectiveness due to the drug resistance evolved in the parasite [3].

The literature on the mathematical model for vectorborne disease likewise malaria is vast. The first published model demonstrating the life cycle of the malaria parasite was developed by Sir Ross [4]. The model proposed by Sir Ronald Ross is one of the simplest models, known as the classical Ross model in the literature. It demonstrates the crosstalk between the number of mosquitoes and the proportion of bite that produced infection in the human body. 
This model was frequently used in the past due to its simplicity. However, the simplicity of the mathematical model is at the cost of limitations in the analysis. Therefore, considering the great challenge of malaria disease, several researchers have developed/modified existing models by introducing different factors/parameters in the model.

The mathematical modeling of infectious disease has proved to play an important role in understanding the insights of the transmission dynamics and appropriate control strategies [5]. In past, several mathematical models have been proposed to analyze this deadly disease. The extension of Sir Ross's model includes the modification/addition of different important factors likewise, latent period of infection [6], immunity factor [7], the heterogeneity of human and mosquito $[8,9]$, susceptibility to malaria in host population $[10,11]$, exposed human and mosquito $[3,12]$, and recovered human $[8,13]$, among others.

Macdonald has introduced the effect of exposed mosquitoes by employing a separate differential equation catering the rate of change of exposed mosquitoes [7]. Similarly, Anderson and May proposed to add the rate of change of exposed human in the model [6]. The comparison of two epidemiological models of immunity to malaria shows that different characterizations of immunity, boosted by exposure to infection, generate qualitatively different results [8]. Nagwa and Shu analyzed the deterministic differential equation model for endemic malaria in the presence of the variable host and parasite population [13]. Their results suggested that disease is persistent if the threshold parameter exceeds the barrier of magnitude unity; otherwise, a disease-free equilibrium always exists. In a similar study, Chitnis and co-authors have presented the bifurcation analysis of the reproductive number (RN) [14]. RN is the number of secondary infections that one infectious individual would create over the time course of the disease period, provided that total population except infectious is susceptible [15]. Lashari and co-authors formulated the vector-borne disease environment in the form of an optimal control problem. They have introduced three different control parameters including personal protection, disease medical treatment, and mosquito reduction strategies [3]. Ozair and co-authors have analyzed the transmission dynamics of malaria disease with a nonlinear incident rate [16]. Addawe and Lope proposed to divide the human population into two compartments: preschool ( $0-5$ years) and over five age [17]. Their findings verify the existing results that asymptotical stability is guaranteed with $\mathrm{RN}$ magnitude less than unity. Mishra and co-authors, Samanta and co-authors, and Greenhalag and co-authors have proposed and analyzed a nonlinear mathematical model to assess the effect of awareness by media on the prevalence of infectious disease [18-20]. Cai et al. introduced a malaria model with an asymptomatic class in human population and exposed classes in both human and vector populations [21]. Sung Chan and co-authors have studied the vector-bias mathematical model and considered two different incidence areas: a high transmission area and a low transmission area [22]. Recently, Sung Chan and co-authors have developed a new transmission model to evaluate the rate of malaria relapse infections in the northern part of Korea and to examine its effect at the population-level on radical cure [23].

In this article, a deterministic vector-borne disease model is proposed. Previous studies suggested that prevention is a control parameter for such infectious diseases. Thus, it shall be helpful to add awareness terms in the mathematical model of the disease. The whole infected host population is divided into two groups, aware and unaware infected individuals. We analyzed the model to study the impact of awareness programs conducted by awareness campaign through medical staff on the spread of malaria disease. In the modeling process, it is assumed that the growth rate of the cumulative density of awareness program will increase with an increase in the unaware infected individuals in the host population. We also assumed that both infected classes can spread disease when the mosquitoes contact them, but the aware class has very low chance to spread disease due to awareness campaigns. It is further assumed that due to awareness, the contact rate of infected mosquito interaction with aware humans will be reduced. The performance of the proposed model is evaluated by comparing the result with previous models. The result of the proposed model suggests that disease-free equilibrium is achieved earlier than the existing model.

The rest of the article is as follows. In section 2 , the formulation of a mathematical model is presented. Section 3 describes the positivity and boundedness of solution. In section 4 , the existence of disease-free equilibrium including derivation of basic reproduction number and stability analysis of the model is presented. The results of numerical simulation are illustrated in Section 5, Section 6 provides discussion, and Section 7 provides conlclusion.

\section{Model Formulation}

Mosquito-borne diseases, e.g., yellow fever, dengue fever, and malaria, are frequently observed in tropical and subtropical countries. These illnesses spread widely in a short period with the life-threatening impact of many human lives. Among these vector-borne diseases, malaria is one of the serious and major illnesses caused by several species of mosquito-borne parasite (Plasmodium falciparum, Plasmodium malaria, Plasmodium ovale, Plasmodium vivax, etc.) [24]. Anopheles female mosquito is responsible for the transmission of disease to the human body through a bite [3]. This blood meal of mosquito converts a healthy human being (susceptible) into a category called infected hosts. The human population that are not infected but under the threat that they can catch malaria infection are known as the exposed population. Individuals recovered from the infected population through medical treatment without threat to their life fall in the group of recovered ones. Figure 1 shows the schematic diagram of the proposed malaria disease model for particular population of humans and mosquitoes.

The human population is divided into five different compartments (susceptible, exposed, unaware infected, aware infected, and recovered) representing the total population at any time $t$. Let $N_{h}$ be the total population in a region under consideration for malaria disease analysis. 


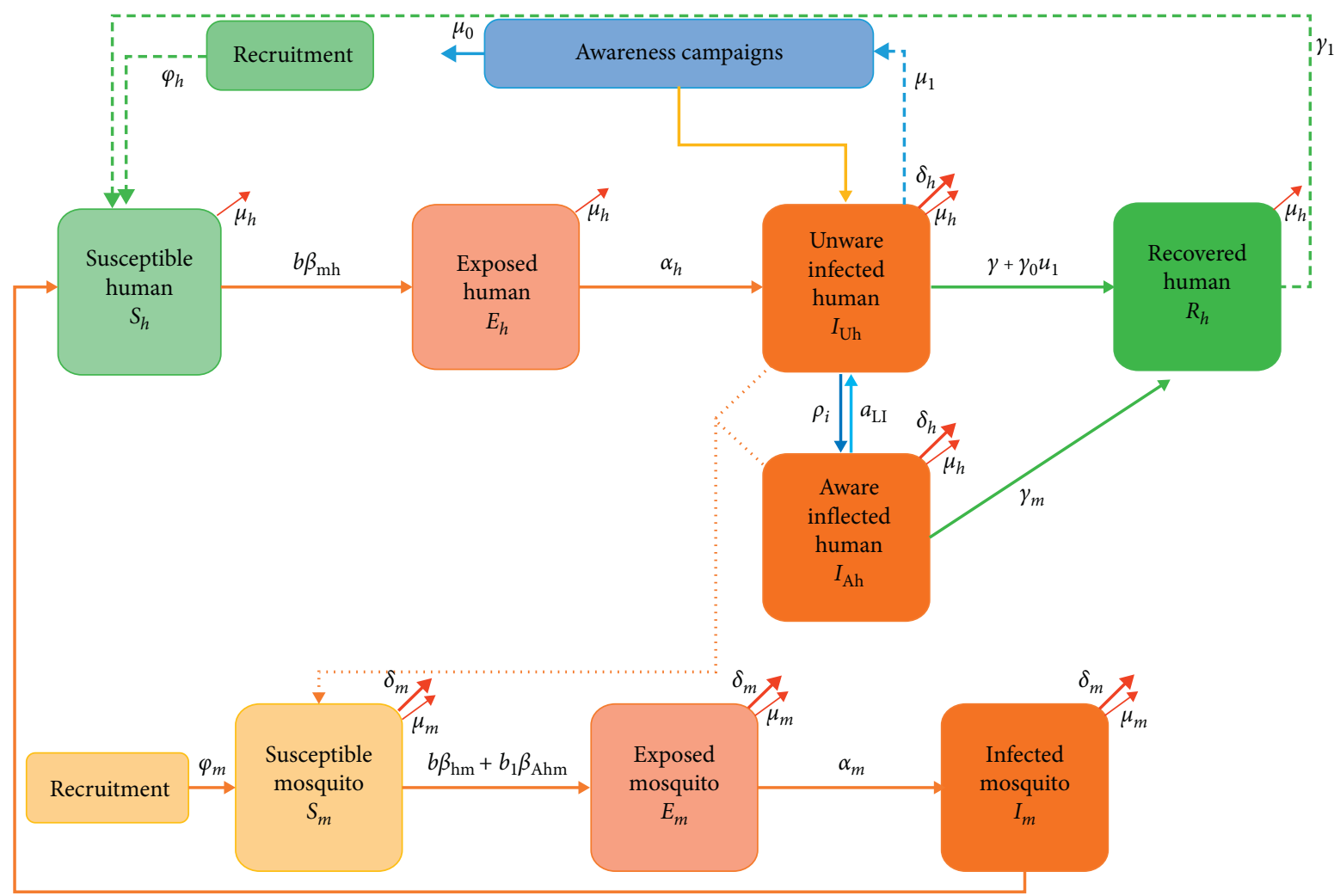

FIGURE 1: Schematic diagram of the proposed model algorithm.

Suppose $S_{h}(t)$ denotes the numbers of humans susceptible to disease, $E_{h}(t)$ is the count for the exposed hosts to disease, $I_{\mathrm{uh}}(t)$ and $I_{\mathrm{Ah}}(t)$ are showing unaware and aware infected human population, and $R_{h}(t)$ is the numbers of individual who have temporarily recovered from disease. Similarly, mosquito population has been grouped into three compartments. Let $S_{m}(t)$ is the count for susceptible mosquito population, $E_{m}(t)$ is the numbers for exposed mosquito population, $I_{m}(t)$ represents the numbers of an infected member of the mosquito population, and $N_{m}(t)$ is the total count of the mosquito population in a particular area of interest. Also, let $M(t)$ be the cumulative density of awareness programs driven in the region at time $t$. The growth rate of the density of awareness programs is assumed to be proportional to unaware infected individuals. It is assumed that the contact of infected mosquitoes with aware individuals will be reduced. The constant $\mu_{1}$ represents the rate at which awareness campaigns are being implemented and $\mu_{0}$ represents the depletion rate of these campaigns due to ineffectiveness, a social problem in the population.

For host population, $\varphi_{h}$ is the recruitment rate of human population in a particular region, $b$ is the contact rate of mosquito to human population, $\beta_{\mathrm{mh}}$ is the probability that bite of infectious mosquito result in the transmission of disease to susceptible human, $\gamma_{1}$ is the rate of loss of immunity in recovered human, $\alpha_{h}$ is the rate of progression from exposed to unaware infected class, $\gamma$ is the treatment rate in the region, $\gamma_{0}$ is the recovery rate of unaware infected population with temporary immunity, $u_{1}$ represents the successful efforts of treatment resulting recovered humans, $\rho_{i}$ is the rate of awareness to unaware infected human, $a_{\mathrm{LI}}$ is the rate of loss of awareness of aware infected human, $\gamma_{m}$ is the recovery rate of aware infected human (that rate is greater than the normal recovery rate due to awareness), and $\delta_{h}$ and $\mu_{h}$ are the disease and natural death rate of the human, respectively.

For mosquito population, $\varphi_{m}$ and $\mu_{m}$ are the mosquito recruitment and natural death rate, $\delta_{m}$ represents the strategies to kill mosquito after awareness, $\beta_{\mathrm{hm}}$ is the probability that a blood meal of an infectious mosquito result in the transmission of disease to susceptible individual, $\beta_{\mathrm{Ahm}}$ is the probability of disease transmission from aware infected human to susceptible mosquitoes, $b_{1}$ is the contact rate between unaware infected human to susceptible mosquitoes, and $\alpha_{m}$ is the rate of progression from exposed mosquitoes to infected mosquitoes. The definitions of the mathematical parameters with their values are summarized in Table 1.

These definitions lead to a set of coupled nonlinear differential equations describing the proposed model, for the infectious vector-borne disease. The mathematical form of the model is described as follows:

$$
\begin{aligned}
& \frac{\mathrm{d} S_{h}(t)}{\mathrm{d} t}=\varphi_{h}-b \beta_{\mathrm{mh}} S_{h}(t) I_{m}(t)+\gamma_{1} R_{h}(t)-\mu_{h} S_{h}(t), \\
& \frac{\mathrm{d} E_{h}(t)}{\mathrm{d} t}=b \beta_{\mathrm{mh}} S_{h}(t) I_{m}(t)-\left(\alpha_{h}+\mu_{h}\right) E_{h}(t),
\end{aligned}
$$


TABLE 1: Definition and values of parameters used in the model.

\begin{tabular}{|c|c|c|c|}
\hline Parameters & Definition & Values & References \\
\hline$\varphi_{h}$ & Recruitment rate of human & 10 & {$[3]$} \\
\hline$\gamma_{1}$ & Rate of loss of immunity to recovered human & $1 / 730$ & {$[25]$} \\
\hline$\mu_{h}$ & Natural death rate of human & $1 / 60 * 365$ & {$[3]$} \\
\hline$b$ & Contact rate between mosquito and human & 3 & {$[3]$} \\
\hline$\beta_{\mathrm{mh}}$ & Disease transmission rate from mosquito to human & 0.001 & [3] \\
\hline$\alpha_{h}$ & Rate of progression from exposed to unaware infected & $1 / 17$ & {$[3]$} \\
\hline$\gamma$ & Treatment rate in the region & 0.07 & [3] \\
\hline$\gamma_{0}$ & Recovery rate of unaware infected human & 0.04 & {$[3]$} \\
\hline$u_{1}$ & Successful effort of treatment ( 0 or 1$)$ & 1 & \\
\hline$\rho_{i}$ & Rate of awareness to unaware infected human & 0.0005 & Assumed \\
\hline$\delta_{h}$ & Disease death rate of infected human & 0.01 & [3] \\
\hline$a_{L I}$ & Rate of loss of awareness to uninfected human & 0.02 & Assumed \\
\hline$\gamma_{m}$ & Recovery rate of aware infected human & 0.012 & Assumed \\
\hline$\varphi_{m}$ & Recruitment rate of mosquito & 50 & [3] \\
\hline$\beta_{\mathrm{uhm}}$ & Disease transmission rate from unaware human to mosquito & 0.0001 & [3] \\
\hline$b_{1}$ & Contact rate between mosquito and aware human & 0.12 & Assumed \\
\hline$\beta_{\mathrm{Ahm}}$ & Disease transmission rate from aware human to mosquito & 0.0001 & Assumed \\
\hline$\mu_{m}$ & Natural death rate of mosquito & $1 / 15$ & [3] \\
\hline$\delta_{m}$ & Death rate of mosquito due to human awareness & $1 / 50$ & Assumed \\
\hline$\alpha_{m}$ & Rate of progression from exposed mosquito to infected mosquito & $1 / 18$ & [3] \\
\hline$\mu_{1}$ & Constant rate influenced by unaware infected human & 0.02 & Assumed \\
\hline$\mu_{0}$ & Depletion rate of awareness & 0.01 & Assumed \\
\hline
\end{tabular}

$$
\begin{aligned}
\frac{\mathrm{d} I_{\mathrm{Uh}}(t)}{\mathrm{d} t}= & \alpha_{h} E_{h}(t)-\left(\delta_{h}+\mu_{h}+\gamma+\gamma_{0} u_{1}+\rho_{i} M(t)\right) I_{\mathrm{Uh}}(t) \\
& +a_{\mathrm{LI}} I_{\mathrm{Ah}}(t)
\end{aligned}
$$

$$
\begin{aligned}
\frac{\mathrm{d} I_{\mathrm{Ah}}(t)}{\mathrm{d} t}= & \rho_{i} M(t) I_{\mathrm{Uh}}(t)-\left(\mu_{h}+\delta_{h}\right) I_{\mathrm{Ah}}(t)-\gamma_{m} I_{\mathrm{Ah}}(t) \\
& -a_{\mathrm{LI}} I_{\mathrm{Ah}}(t),
\end{aligned}
$$

$\frac{\mathrm{d} R_{h}(t)}{\mathrm{d} t}=\left(\gamma+\gamma_{0} u_{1}\right) I_{\mathrm{Uh}}(t)+\gamma_{m} I_{\mathrm{Ah}}(t)-\left(\mu_{h}+\gamma_{1}\right) R_{h}(t)$,

$$
\begin{aligned}
\frac{\mathrm{d} S_{m}(t)}{\mathrm{d} t}= & \varphi_{m}-\left(\mu_{m}+\delta_{m}\right) S_{m}(t)-b \beta_{\mathrm{hm}} S_{m}(t) I_{\mathrm{Uh}}(t) \\
& -b_{1} \beta_{\mathrm{Ahm}} S_{m}(t) I_{\mathrm{Ah}}(t),
\end{aligned}
$$

$$
\begin{aligned}
\frac{\mathrm{d} E_{m}(t)}{\mathrm{d} t}= & b \beta_{\mathrm{hm}} S_{m}(t) I_{\mathrm{Uh}}(t)+b_{1} \beta_{\mathrm{Ahm}} S_{m}(t) I_{\mathrm{Ah}}(t) \\
& -\left(\mu_{m}+\delta_{m}+\alpha_{m}\right) E_{m}(t), \\
\frac{\mathrm{d} I_{m}(t)}{\mathrm{d} t}= & \alpha_{m} E_{m}(t)-\left(\mu_{m}+\delta_{m}\right) I_{m}(t), \\
\frac{\mathrm{d} M(t)}{\mathrm{d} t}= & \mu_{1} I_{\mathrm{Uh}}(t)-\mu_{0} M(t) .
\end{aligned}
$$

\section{Positivity and Boundedness of Solutions}

The mathematical model presented in the system of equations (1)-(9) describes the rate of change of different compartments of human and mosquito population. Therefore, it is important to verify that all solutions with nonnegative initial conditions shall remain nonnegative for all time. All the solutions of proposed system, which initiates inside region $D$, remain in region $D$. Mathematically,

This result can be summarized in the following theorem.

Theorem 1. For all time $t \geq 0$, there exists a domain:

$$
D=\left\{\begin{array}{c}
\left(S_{h}(t), E_{h}(t), I_{\mathrm{Uh}}(t), I_{\mathrm{Ah}}(t), R_{h}(t), S_{m}(t), E_{m}(t), I_{m}(t)\right) \in R_{+}^{8}: S_{h}(0)>0, E_{h}(0) \geq 0 \\
I_{\mathrm{Uh}}(0) \geq 0, I_{\mathrm{Ah}}(0) \geq 0, R_{h}(0) \geq 0, S_{m}(0) \geq 0, E_{m}(0) \geq 0, I_{m}(0) \geq 0 ; N_{h} \leq\left(\varphi_{h} / \mu_{h}\right) \\
N_{m} \leq\left(\varphi_{m} / \mu_{m}\right)
\end{array}\right\} .
$$

All the solutions of the system of equations (1)-(9) are bounded in domain D:
Proof. Let $\quad\left(S_{h}(t), E_{h}(t), I_{U h}(t), I_{A h}(t), R_{h}(t), S_{m}(t)\right.$, $\left.E_{m}(t), I_{m}(t)\right)$ be any solution with positive initial conditions 
$S_{h}(0)>\quad 0, E_{h}(0) \geq 0, I_{\mathrm{Uh}}(0) \geq 0, \quad I_{\mathrm{Ah}}(0) \geq 0, R_{h}(0) \geq 0, S_{m}$ $(0) \geq 0, E_{m}(0) \geq 0, I_{m}(0) \geq 0$, with $N_{h}\left(S_{h}(t), E_{h}(t), I_{\mathrm{Uh}}(t)\right.$, $\left.I_{\mathrm{Ah}}(t), R_{h}(t)\right)=S_{h}(t)+E_{h}(t)+I_{\mathrm{Uh}}(t)+I_{\mathrm{Ah}}(t)+R_{h}(t)$ and $N_{m}\left(S_{m}(t), E_{m}(t), I_{m}(t)\right)=S_{m}(t)+E_{m}(t)+I_{m}(t)+E_{m}(t)$ $+I_{m}(t)$. Notice that the sum of the first five compartments $S_{h}(t), E_{h}(t), I_{\mathrm{Uh}}(t), I_{\mathrm{Ah}}(t), R_{h}(t)$ in system of equations (1)-(9) is equal to the total human population of size $N_{h}$ and the sum of compartments $\left(S_{m}(t), E_{m}(t), I_{m}(t)\right)$ is equal to the total mosquito population of size $N_{m}$. Hence adding these equations yields the time derivative along with the solution of equations (1)-(9) given as

$$
\begin{aligned}
& \frac{\mathrm{d} N_{h}}{\mathrm{~d} t}=\varphi_{h}-\mu_{h} N_{h}-\delta_{h}\left(I_{\mathrm{Uh}}+I_{\mathrm{Ah}}\right) \leq \varphi_{h}-\mu_{h} N_{h} . \\
& \frac{\mathrm{d} N_{m}}{\mathrm{~d} t}=\varphi_{m}-\mu_{m} N_{m}-\delta_{m} N_{m} \leq \varphi_{m}-\mu_{m} N_{m} .
\end{aligned}
$$

Equations (11) and (12) can be written as

$$
\begin{gathered}
\left(\frac{\mathrm{d} N_{h}}{\mathrm{~d} t}\right)+\mu_{h} N_{h} \leq \varphi_{h}, \\
\left(\frac{\mathrm{d} N_{m}}{\mathrm{~d} t}\right)+\mu_{m} N_{m} \leq \varphi_{m} .
\end{gathered}
$$

Solving these different inequalities yields

$$
\begin{aligned}
& N_{h} \leq\left(\frac{\varphi_{h}}{\mu_{h}}\right)\left(1-e^{-\mu_{h} t}\right)+N_{h}(0) e^{-\mu_{h} t}, \\
& N_{m} \leq\left(\frac{\varphi_{m}}{\mu_{m}}\right)\left(1-e^{-\mu_{m} t}\right)+N_{m}(0) e^{-\mu_{m} t} .
\end{aligned}
$$

Consequently, taking the limit as $t \longrightarrow \infty$ gives $N_{h} \leq\left(\varphi_{h} / \mu_{h}\right) \& N_{m} \leq\left(\varphi_{m} / \mu_{m}\right)$. Thus, $D$ is positively invariant, and all the solutions are bounded in the interval $[0, \infty)$.

\section{Existence of Disease-Free Equilibrium Point}

Disease-free equilibrium points are the steady-state solution, when there is no malaria infection. Thus, the disease-free equilibrium point for the system of equations (1)-(9) implies that $E_{h}=0, I_{\mathrm{uh}}=0, I_{\mathrm{Ah}}=0, \quad R_{h}=0, E_{m}=0$, and $I_{m}=0$, and after solving the equations (1) and (6) of system yields $S_{h}=\left(\varphi_{h} / \mu_{h}\right)$ and $S_{m}=\left(\varphi_{m} / \mu_{m}+\delta_{m}\right)$. Thus, we obtain the disease-free equilibrium point, $E_{1}$ :

$$
E_{1}=\left(\left(\frac{\varphi_{h}}{\mu_{h}}\right), 0,0,0,0,\left(\frac{\varphi_{m}}{\mu_{m}+\delta_{m}}\right), 0,0,0\right) .
$$

4.1. Basic Reproductive Number. The basic reproductive number $R_{0}$ measures the average number of new malaria infections generated by a single infected individual in a completely susceptible population [26].

To obtain $R_{0}$ for system of equations (1)-(9), we used the next-generation matrix technique described in $[15,25,27,28]$. Let $x=\left(E_{h}, I_{\mathrm{Uh}}, I_{\mathrm{Ah}}, E_{m}, I_{m}, S_{h}, R_{h}, S_{m}\right)^{T}$, then the model system of equations (1)-(9) can be written as

$$
\frac{\mathrm{d} x}{\mathrm{~d} t}=F(x)-V(x)
$$

where

$$
V(x)=\left(V^{-}(x)-V^{+}(x)\right)
$$

$$
F(x)=\left(\begin{array}{c}
b \beta_{\mathrm{mh}} S_{h} I_{m} \\
0 \\
0 \\
b \beta_{\mathrm{hm}} S_{m} I_{\mathrm{Uh}}+b_{1} \beta_{\mathrm{Ahm}} S_{m} I_{\mathrm{Ah}} \\
0 \\
0 \\
0 \\
0 \\
0 \\
-\alpha_{h} E_{h}-a_{\mathrm{LI}} I_{\mathrm{Ah}}+\left(\delta_{h}+\mu_{h}+\gamma+\gamma_{0} u_{1}+\rho_{i} M\right) I_{\mathrm{Uh}} \\
-\rho_{i} M I_{\mathrm{Uh}}+\left(\mu_{h}+\delta_{h}+\gamma_{m}+a_{\mathrm{LI}}\right) I_{\mathrm{Ah}} \\
\left(\mu_{m}+\delta_{m}+\alpha_{m}\right) E_{m} \\
-\alpha_{m} E_{m}+\left(\mu_{m}+\delta_{m}\right) I_{m} \\
-\varphi_{h}-\gamma_{1} R_{h}+b \beta_{\mathrm{mh}} S_{h} I_{m}+\mu_{h} S_{h} \\
-\left(\gamma+\gamma_{0} u_{1}\right) I_{\mathrm{Uh}}-\gamma_{m} I_{\mathrm{Ah}}+\left(\mu_{h}+\gamma_{1}\right) R_{h} \\
-\varphi_{m}+\left(\mu_{m}+\delta_{m}\right) S_{m}+b \beta_{\mathrm{hm}} S_{m} I_{\mathrm{Uh}}+b_{1} \beta_{\mathrm{Ahm}} S_{m} I_{\mathrm{Ah}} \\
-\mu_{1} I_{\mathrm{Uh}}+\mu_{0} M
\end{array}\right)
$$

Finding the partial derivative of $F$ and $V$ at disease-free equilibrium point $E_{1}$ gives $F$ and $V$, respectively, as follows:

$$
F=\left(\begin{array}{ccccc}
0 & 0 & 0 & 0 & b \beta_{\mathrm{mh}}\left(\varphi_{h} / \varphi_{h}\right) \\
0 & 0 & 0 & 0 & 0 \\
0 & 0 & 0 & 0 & 0 \\
0 & b \beta_{\mathrm{hm}}\left(\varphi_{m} / \mu_{m}+\delta_{m}\right) & b_{1} \beta_{\mathrm{Ahm}}\left(\varphi_{m} / \mu_{m}+\delta_{m}\right) & 0 & 0 \\
0 & 0 & 0 & 0 & 0
\end{array}\right) \text {, }
$$




$$
V=\left(\begin{array}{ccccc}
\alpha_{h}+\mu_{h} & 0 & 0 & 0 & 0 \\
-\alpha_{h} & \delta_{h}+\mu_{h}+\gamma+\gamma_{0} u_{1} & -a_{\mathrm{LI}} & 0 & 0 \\
0 & 0 & \mu_{h}+\delta_{h}+\gamma_{m}+a_{\mathrm{LI}} & 0 & 0 \\
0 & 0 & 0 & \mu_{m}+\delta_{m}+\alpha_{m} & 0 \\
0 & 0 & 0 & -\alpha_{m} & \mu_{m}+\delta_{m}
\end{array}\right) .
$$

So that

$$
V^{-1}=\left(\begin{array}{ccccc}
v_{11}^{-1} & 0 & 0 & 0 & 0 \\
v_{21}^{-1} & v_{22}^{-1} & v_{23}^{-1} & 0 & 0 \\
0 & 0 & v_{33}^{-1} & 0 & 0 \\
0 & 0 & 0 & v_{44}^{-1} & 0 \\
0 & 0 & 0 & v_{54}^{-1} & v_{55}^{-1}
\end{array}\right),
$$

where $v_{11}^{-} 1=\left(1 / \alpha_{h}+\mu_{h}\right), v_{21}^{-1}=\left(-\alpha_{h} /\left(\alpha_{h}+\mu_{h}\right)\left(\delta_{h}+\mu_{h}+\right.\right.$ $\left.\left.\gamma+\gamma_{0} u_{1}\right)\right), \quad v_{22}^{-1}=\left(1 / \delta_{h}+\mu_{h}+\gamma+\gamma_{0} u_{1}\right), v_{23}^{-1}=\left(-a_{\mathrm{LI}} /\left(\delta_{h}+\right.\right.$ $\left.\left.\mu_{h}+\gamma+\gamma_{0} u_{1}\right)\left(\mu_{h}+\delta_{h}+\gamma_{m}+a_{L I}\right)\right), v_{33}^{-1}=\left(1 / \mu_{h}+\delta_{h}+\gamma_{m}+\right.$ $\left.a_{\mathrm{LI}}\right), v_{44}^{-1}=\left(1 / \mu_{m}+\delta_{m}+\alpha_{m}\right), \quad v_{54}^{-1}=\left(-\alpha_{m} /\left(\mu_{m}+\delta_{m}+\alpha_{m}\right)\right.$ $\left.\left(\mu_{m}+\delta_{m}\right)\right), v_{55}^{-1}=\left(1 / \mu_{m}+\delta_{m}\right)$.

The basic reproductive number $R_{0}=\rho\left(\mathrm{FV}^{-1}\right)$ is the spectral radius of the product $\mathrm{FV}^{-1}$. Thus,

$$
R_{0}=\rho\left(\mathrm{FV}^{-1}\right)=\sqrt{\frac{\varphi_{h} \varphi_{m} \alpha_{h} \alpha_{m} b^{2} \beta_{\mathrm{mh}} \beta_{\mathrm{hm}}}{\mu_{h}\left(\alpha_{h}+\mu_{h}\right)\left(\delta_{h}+\mu_{h}+\gamma+\gamma_{0} u_{1}\right)\left(\mu_{m}+\delta_{m}+\alpha_{m}\right)\left(\mu_{m}+\delta_{m}\right)^{2}}} .
$$

In equation (25), $\left(\alpha_{h} /\left(\alpha_{h}+\mu_{h}\right)\right)$ is the probability that a human will survive the exposed state to become infectious; $\left(1 /\left(\delta_{h}+\mu_{h}+\gamma+\gamma_{0} u_{1}\right)\right)$ is the average duration of the infectious period of the human; $\left(\alpha_{m} /\left(\mu_{m}+\delta_{m}+\alpha_{m}\right)\right)$ is the probability that a mosquito will survive the exposed state to become infectious; and $\left(1 /\left(\mu_{m}+\delta_{m}\right)\right)$ is the state to become infectious. Let the basic reproductive number, $R_{0}$, be written as $R_{0}=\sqrt{R_{h} R_{m}}$, where $R_{h}=\left(\varphi_{h} \alpha_{h} b \beta_{\mathrm{mh}} / \mu_{h}\left(\alpha_{h}+\mu_{h}\right)\left(\delta_{h}+\right.\right.$ $\left.\left.\mu_{h}+\gamma+\gamma_{0} u_{1}\right)\right)$ and $R_{m}=\left(\varphi_{m} \alpha_{m} b \beta_{\mathrm{hm}} /\left(\mu_{m}+\delta_{m}+\alpha_{m}\right)\right.$ $\left.\left(\mu_{m}+\delta_{m}\right)^{2}\right)$.

Here, $R_{h}$ describes the number of humans that one infectious mosquito infects over its expected infection period in a completely susceptible human population and $R_{m}$ describes the number of mosquitoes infected by one infectious human during the period of infectiousness in a completely susceptible mosquito population.

4.2. Stability Analysis of Disease-Free Equilibrium. We analyzed the stability of disease-free equilibrium of the system of equations (1)-(9) by using the basic reproductive number $R_{0}$ in the following theorem.

Theorem 2. The disease-free equilibrium (DFE) $E_{1}$ is locally asymptotically stable if $R_{0} \leq 1$ and unstable if $R_{0}>1$.
Proof. The Jacobian of the system of equations (1)-(9) evaluated at the disease-free equilibrium point $E_{1}$ is obtained as follows:

$$
J\left(E_{1}\right)=\left[\begin{array}{ccccccccc}
j_{11} & 0 & 0 & 0 & j_{15} & 0 & 0 & j_{18} & 0 \\
0 & j_{22} & 0 & 0 & 0 & 0 & 0 & j_{28} & 0 \\
0 & j_{32} & j_{33} & j_{34} & 0 & 0 & 0 & 0 & 0 \\
0 & 0 & 0 & j_{44} & 0 & 0 & 0 & 0 & 0 \\
0 & 0 & 0 & j_{54} & j_{55} & 0 & 0 & 0 & 0 \\
0 & 0 & j_{63} & j_{64} & j_{66} & j_{66} & 0 & 0 & 0 \\
0 & 0 & j_{73} & j_{74} & 0 & 0 & j_{77} & 0 & 0 \\
0 & 0 & 0 & 0 & 0 & 0 & j_{87} & j_{88} & 0 \\
0 & 0 & j_{93} & 0 & 0 & 0 & 0 & 0 & j_{99}
\end{array}\right] \text {, }
$$

where $j_{11}=-\mu_{h}, j_{15}=\gamma_{1}, j_{18}=-b \beta_{\mathrm{mh}}\left(\varphi_{h} / \mu_{h}\right), j_{22}=-\left(\alpha_{h}+\right.$ $\left.\mu_{h}\right), j_{28}=b \beta_{\mathrm{mh}}\left(\varphi_{h} / \mu_{h}\right), j_{32}=\alpha_{h}, j_{33}=-\left(\delta_{h}+\mu_{h}+\gamma+\gamma_{0} u_{1}\right)$, $j_{34}=a_{\mathrm{LI}}, j_{44}=-\left(\mu_{m}+\gamma_{m}+\delta_{m}+a_{L I}\right), j_{54}=\gamma_{m}, j_{55}=-\left(\mu_{h}\right.$ $\left.+\gamma_{1}\right), j_{63}=-b \beta_{\mathrm{hm}}\left(\varphi_{m} / \mu_{m}+\delta_{m}\right), \quad j_{64}=-b_{1} \beta_{\mathrm{Ahm}}\left(\varphi_{m} / \mu_{m}+\right.$ $\left.\delta_{m}\right), \quad j_{66}=-\left(\mu_{m}+\delta_{m}\right), j_{73}=b \beta_{\mathrm{hm}}\left(\varphi_{m} / \mu_{m}+\delta_{m}\right), j_{74}=b_{1}$ $\beta_{\mathrm{Ahm}}\left(\varphi_{m} / \mu_{m}+\delta_{m}\right), \quad j_{77}=-\left(\mu_{m}+\delta_{m}+\alpha_{m}\right), j_{87}=\alpha_{m}, j_{88}=$ $-\left(\mu_{m}+\delta_{m}\right), j_{93}=\mu_{1}, j_{99}=-\mu_{0}$. 
The DFE, $E_{1}$, is locally asymptotically stable (LAS) if we show that all the eigenvalues of equation (26) have negative real part. Since the first, sixth, and ninth columns of equation (26) contain only the diagonal terms, three eigenvalues $-\mu_{h},-\left(\mu_{m}+\delta_{m}\right)$, and $-\mu_{0}$ can be obtained from these columns. Remaining eigenvalues of equation (26) can be obtained from the submatrix $J_{1}$ formed by excluding the first, sixth, and ninth rows and columns of equation (26). Hence, we have

$$
J_{1}\left(E_{1}\right)=\left[\begin{array}{cccccc}
j_{22} & 0 & 0 & 0 & 0 & j_{28} \\
j_{32} & j_{33} & 0 & 0 & 0 & 0 \\
0 & 0 & j_{44} & 0 & 0 & 0 \\
0 & 0 & j_{54} & j_{55} & 0 & 0 \\
0 & j_{73} & j_{74} & 0 & j_{77} & 0 \\
0 & 0 & 0 & 0 & j_{87} & j_{88}
\end{array}\right] .
$$

Similarly, the fourth column of equation (27) contains only the diagonal term which forms negative eigenvalues $-\left(\mu_{h}+\gamma_{1}\right)$. All the remaining eigenvalues of equation (27) can be calculated by the submatrix $J_{2}$ :

$$
J_{2}\left(E_{1}\right)=\left[\begin{array}{ccccc}
j_{22} & 0 & 0 & 0 & j_{28} \\
j_{32} & j_{33} & 0 & 0 & 0 \\
0 & 0 & j_{44} & 0 & 0 \\
0 & j_{73} & j_{74} & j_{77} & 0 \\
0 & 0 & 0 & j_{87} & j_{88}
\end{array}\right] \text {. }
$$

The eigenvalues of equation (28) are the roots of the following characteristic equation:

$$
\left(\lambda-j_{44}\right)\left(\left(\lambda-j_{22}\right)\left(\lambda-j_{33}\right)\left(\lambda-j_{77}\right)\left(\lambda-j_{88}\right)-j_{28} j_{32} j_{73} j_{87}\right)=0 .
$$

From equation (29), we can get five eigenvalues. One of the eigenvalues $j_{44}=-\left(\mu_{m}+\gamma_{m}+\delta_{m}+a_{\mathrm{LI}}\right)$ has a negative real part. The other four eigenvalues can be obtained from equation (30):

$$
\left.\begin{array}{l}
\left.\begin{array}{l}
\left(\lambda-j_{22}\right)\left(\lambda-j_{33}\right)\left(\lambda-j_{77}\right)\left(\lambda-j_{88}\right)-j_{28} j_{32} j_{73} j_{87}=0 \\
\left(\lambda+\alpha_{h}+\mu_{h}\right)\left(\lambda+\delta_{h}+\mu_{h}+\gamma+\gamma_{0} u_{1}\right)\left(\lambda+\mu_{m}+\delta_{m}+\alpha_{m}\right)\left(\lambda+\mu_{m}+\delta_{m}\right) \\
-\frac{b^{2} \alpha_{h} \alpha_{m} \beta_{\mathrm{mh}} \beta_{\mathrm{hm}} \varphi_{h} \varphi_{m}}{\mu_{h}\left(\mu_{m}+\delta_{m}\right)}
\end{array}\right\}=0 \\
b
\end{array}\right\} .
$$

If we let $b_{1}=-j_{22}, b_{2}=-j_{33}, b_{3}=-j_{77}$, and $b_{1}=-j_{88}$, then equation (30) becomes

where

$$
\left.\begin{array}{l}
a_{4}=1, a_{3}=b_{1}+b_{2}+b_{3}+b_{4}, a_{2}=b_{1} b_{2}+b_{1} b_{3}+b_{1} b_{4}+b_{2} b_{3}+b_{2} b_{4}+b_{3} b_{4}, \\
a_{1}=b_{1} b_{2} b_{3}+b_{1} b_{2} b_{4}+b_{1} b_{3} b_{4}+b_{2} b_{3} b_{4}, a_{0}=b_{1} b_{2} b_{3} b_{4}-\frac{b^{2} \alpha_{h} \alpha_{m} \varphi_{h} \varphi_{m} \beta_{\mathrm{mh}} \beta_{\mathrm{hm}}}{\mu_{h}\left(\mu_{m}+\delta_{m}\right)},
\end{array}\right\}
$$

where $a_{0}$ is in the terms of reproduction number and $R_{0}$, can be written as

$$
a_{0}=b_{1} b_{2} b_{3} b_{4}\left(1-R_{0}\right) .
$$

We employ the Routh-Hurwitz criterion, which states that all the roots of equation (31), has negative real parts, if and only if the coefficients $a_{i}$ are positive and matrices $H_{i}>0$ for $i=0,1,2,3$, and 4 . From equation (32), it is easy to see that $a_{1}>0, a_{2}>0, a_{3}>0$, and $a_{4}>0$ since all $b_{i}^{\prime} s>0$. Moreover, if $R_{0}<1$, it follows from equation (33) that $a_{0}>0$. Also, the Hurwitz matrices for equation (31) are found to be positive that are given as follows:

$$
\begin{aligned}
& H_{1}=a_{1}>0, \\
& H_{2}=\operatorname{det}\left(\begin{array}{ll}
a_{3} & a_{4} \\
a_{1} & a_{2}
\end{array}\right)>0, \\
& H_{3}=\operatorname{det}\left(\begin{array}{lll}
a_{3} & a_{4} & a_{0} \\
a_{1} & a_{2} & a_{3} \\
0 & a_{0} & a_{1}
\end{array}\right)>0, \\
& H_{4}=\operatorname{det}\left(\begin{array}{cccc}
a_{3} & a_{4} & 0 & 0 \\
a_{1} & a_{2} & a_{3} & a_{4} \\
0 & a_{0} & a_{1} & a_{2} \\
0 & 0 & 0 & a_{0}
\end{array}\right)>0
\end{aligned}
$$


Therefore, all the eigenvalues of the Jacobian matrix in equation (26) have negative real parts, when $R_{0}<1$ and DFE is LAS. However, when $R_{0}>1$ we see that $a_{0}<0$ and by Descartes's rule of signs, there is exactly one sign-change in the sequence $a_{4}, a_{3}, a_{2}, a_{1}$, and $a_{0}$ of the coefficient of equation (31). So, there is one eigenvalue with the positive real part and DFE is unstable.

Remark 1. The case for $R_{0}=1$ is possible when $a_{0}=0$ in the characteristic equation. By considering the special case of Routh-Hurwitz stability criterion [29], and replacing this term with very small value, i.e., $\varepsilon$, the result could be interpreted as first case, i.e., $R_{0}<1$.

Theorem 3. The disease-free equilibrium (DFE) of the system of equations (1)-(9) is globally asymptotically stable (GAS) on $D$ if $R_{0}<1$.
Proof. To prove this theorem, we adopt the method described in [30-32]. Castillo-Chavez and co-authors described this method to prove the GAS of DFE in their research article [32]. We begin the proof by defining new variables and breaking our system of equations (1)-(9) into two subsystems. With $X=\left(S_{h}, R_{h}, S_{m}\right)$ and $I=\left(E_{h}, I_{u h}, I_{A h}, E_{m}, I_{m}\right)$, this system can be written as

$$
\begin{aligned}
\frac{\mathrm{d} X}{\mathrm{~d} t} & =F(X, I), \\
\frac{\mathrm{d} I}{\mathrm{~d} t} & =G(X, I) \text { with } G(X, 0)=0,
\end{aligned}
$$

where $X \in R^{3}$ denotes the number of uninfected compartments and $I \in R^{5}$ denotes the number of infected compartments.

The two vector-valued functions $F(X, I)$ and $G(X, I)$ are given as

$$
\begin{gathered}
F(X, I)=\left(\begin{array}{c}
\varphi_{h}-b \beta_{\mathrm{muh}} S_{h}(t) I_{m}(t)+\gamma_{1} R_{\mathrm{Uh}}(t)-\mu_{h} S_{h}(t) \\
\left(\gamma+\gamma_{0} u_{1}\right) I_{\mathrm{Uh}}(t)-\left(\mu_{h}+\gamma_{1}\right) R_{h}(t)+\gamma_{m} I_{\mathrm{Ah}}(t) \\
\varphi_{m}-\left(\mu_{m}+\delta_{m}\right) S_{m}(t)-b \beta_{\mathrm{Uhm}} S_{m}(t) I_{\mathrm{Uh}}(t)-b_{1} \beta_{\mathrm{Ahm}} S_{m}(t) I_{\mathrm{Ah}}(t)
\end{array}\right) \\
b \beta_{\mathrm{mh}} S_{h}(t) I_{m}(t)-\left(\alpha_{h}+\mu_{h}\right) E_{h}(t) \\
G(X, I)=\left(\begin{array}{c}
\alpha_{h} E_{h}(t)-\left(\delta_{h}+\mu_{h}+\gamma+\gamma_{0} u_{1}+\rho_{i} M(t)\right) I_{U h}(t)+a_{L I} I_{\mathrm{Ah}}(t) \\
\rho_{i} M(t) I_{\mathrm{Uh}}(t)-\left(\mu_{h}+\delta_{h}\right) I_{\mathrm{Ah}}(t)-\gamma_{m} I_{\mathrm{Ah}}(t)-a_{\mathrm{LI}} I_{\mathrm{Ah}}(t) \\
b \beta_{\mathrm{Uhm}} S_{m}(t) I_{\mathrm{Uh}}(t)+b_{1} \beta_{\mathrm{Ahm}} S_{m}(t) I_{\mathrm{Ah}}(t)-\left(\mu_{m}+\delta_{m}+\alpha_{m}\right) E_{m}(t) \\
\alpha_{m} E_{m}(t)-\left(\mu_{m}+\delta_{m}\right) I_{m}(t)
\end{array}\right) \text { with } G(X, 0)=0 .
\end{gathered}
$$

$E_{0}=\left(X^{*}, 0\right)$ denotes the disease-free equilibrium of the subsystems, where

$$
X^{*}=\left(\left(\frac{\varphi_{h}}{\mu_{h}}\right), 0,\left(\frac{\varphi_{m}}{\mu_{m}+\delta_{m}}\right)\right) .
$$

The conditions $\left(H_{1}\right)$ and $\left(H_{2}\right)$ below must be met for the global stability:

$$
\left(H_{1}\right): \operatorname{For}\left(\frac{\mathrm{d} X}{\mathrm{~d} t}\right)=F(X, 0), X^{*} \text { is GAS, }
$$

$\left(H_{2}\right): G(X, I)=\mathrm{AI}-\widehat{G}(X, I)$ with $\widehat{G}(X, I) \geq 0, \quad \forall(X, I) \in D$,

where $A$ is an $M$-matrix (the off-diagonal elements of $A$ are nonnegative).

Now consider the reduced system $(\mathrm{d} X / \mathrm{d} t)=F(X, 0)$,

$$
\begin{aligned}
& \frac{\mathrm{d} S_{h}(t)}{\mathrm{d} t}=\varphi_{h}+\gamma_{1} R_{h}(t)-\mu_{h} S_{h}(t), \\
& \frac{\mathrm{d} R_{h}(t)}{\mathrm{d} t}=-\left(\mu_{h}+\gamma_{1}\right) R_{h}(t),
\end{aligned}
$$

$$
\frac{\mathrm{d} S_{m}(t)}{\mathrm{d} t}=\varphi_{m}-\left(\mu_{m}+\delta_{m}\right) S_{m}(t)
$$

$X^{*}=\left(S_{h}, R_{h}, S_{m}\right)=\left(\varphi_{h} / \mu_{h}, 0, \varphi_{m} / \mu_{m}+\delta_{m}\right)$ is the GAS equilibrium point for the reduced system $(\mathrm{d} X / \mathrm{d} t)=F(X, 0)$. To see this, solve equation (43) to obtain $R_{h}=R_{h}(0) e^{-\left(\mu_{h}+\gamma_{1}\right) t} \Longrightarrow R_{h} \longrightarrow 0$ as $t \longrightarrow \infty$. Similarly, from equation (44), we get $S_{m}=\left(\varphi_{m} /\left(\mu_{m}+\delta_{m}\right)\right)+\left(S_{m}(0)-\right.$ $\left.\left(\varphi_{m} /\left(\mu_{m}+\delta_{m}\right)\right)\right) e^{-\left(\mu_{m}+\delta_{m}\right) t} \Longrightarrow S_{m} \longrightarrow\left(\varphi_{m} /\left(\mu_{m}+\delta_{m}\right)\right)$ as $t \longrightarrow \infty$.

Finally, equation (42) yields $S_{h}=\left(\varphi_{h} / \mu_{h}\right)+\left(S_{h}(0)+\right.$ $\left.R_{h}(0)-\left(\varphi_{h} / \mu_{h}\right)\right) e^{-\mu_{h} t} \quad$ and $\quad-R_{h}(0) e^{-\left(\mu_{h}+\gamma_{1}\right) t} \Longrightarrow S_{h} \longrightarrow$ $\left(\varphi_{h} / \mu_{h}\right)$ as $t \longrightarrow \infty$. Hence, the convergence of solutions of equations (42)-(44) is global in $D$. And, $G(X, I)=[\mathrm{AI}-$ $\widehat{G}(X, I)$ with $\widehat{G}(X, I) \geq 0, \forall(X, I) \in D]$, where

$$
A=\left[\begin{array}{ccccc}
-\left(\alpha_{h}+\mu_{h}\right) & 0 & 0 & 0 & \left(\varphi_{h} / \mu_{h}\right) b \beta_{2} \\
\alpha_{h} & -A & a_{\mathrm{LI}} & 0 & 0 \\
0 & \rho_{i} M & -B & 0 & 0 \\
0 & K_{1} b \beta_{2} & K_{1} b_{1} \beta_{3} & -C & 0 \\
0 & 0 & 0 & \alpha_{m} & -D
\end{array}\right] \text {, }
$$


where

$$
\begin{gathered}
\beta_{1}=\beta_{\mathrm{mh}}, \beta_{2}=\beta_{\mathrm{hm}}, \beta_{3}=\beta_{\mathrm{Ahm}}, \quad K_{1}=\left(\Lambda_{m} / \mu_{m}+\delta_{m}\right), \\
A=\mu_{h}+\delta_{h}+\gamma+\gamma_{0} u_{1}+\rho_{i} M,
\end{gathered}
$$

$$
\begin{aligned}
I & =\left[\begin{array}{c}
E_{h} \\
I_{\mathrm{Uh}} \\
I_{\mathrm{Ah}} \\
E_{m} \\
I_{m}
\end{array}\right], \\
\widehat{G}(X, I) & =\left[\begin{array}{c}
b \beta_{2} I_{m}\left(\left(\frac{\varphi_{h}}{\mu_{h}}\right)-S_{h}\right) \\
0 \\
0 \\
\left(b \beta_{2} I_{U h}+b_{1} \beta_{3} I_{A h}\right)\left(\left(\frac{\varphi_{m}}{\mu_{m}+\delta_{m}}\right)-S_{m}\right) \\
0
\end{array}\right] .
\end{aligned}
$$

$$
\begin{aligned}
& S_{h}^{*}=\left(\frac{k_{1} k_{2} k_{3}}{k b \beta_{1} \varphi_{m} \alpha_{m}\left(\mu_{h}+\gamma_{1}\right)\left(k b \beta_{2} I_{\mathrm{UH}}^{*}+b_{1} \beta_{3} \rho_{i} \mu_{1} I_{\mathrm{UH}}^{2}\right)+k k_{2} k_{3} \mu_{h}\left(\mu_{h}+\gamma_{1}\right)}\right), \\
& E_{h}^{*}=\left(\frac{k_{1} b \beta_{1} \varphi_{m} \alpha_{m}\left(k b \beta_{2} I_{\mathrm{UH}}^{*}+b_{1} \beta_{3} \rho_{i} \mu_{1} I_{\mathrm{UH}}^{2}\right)}{k\left(\alpha_{h}+\mu_{h}\right)\left(\mu_{h}+\gamma_{1}\right)\left[b \beta_{1} \varphi_{m} \alpha_{m}\left(k b \beta_{2} I_{\mathrm{UH}}^{*}+b_{1} \beta_{3} \rho_{i} \mu_{1} I_{\mathrm{UH}}^{2}\right)+\mu_{h} k_{2} k_{3}\right]}\right), \\
& I_{\mathrm{Ah}}^{*}=\frac{\mu_{1} \rho_{i} I_{\mathrm{UH}}^{2}}{k}, \\
& R_{h}^{*}=\frac{k\left(\gamma+\gamma_{0} u_{1}\right) I_{\mathrm{UH}}^{*}+\gamma_{m} \rho_{i} \mu_{1} I_{\mathrm{UH}}^{\frac{2}{*}}}{k\left(\mu_{h}+\gamma_{1}\right)}, \\
& S_{m}^{*}=\frac{k \varphi_{m}}{k_{3}}, \\
& E_{m}^{*}=\frac{\left(k b \varphi_{m} \beta_{2} I_{\mathrm{UH}}^{*}+b_{1} \varphi_{m} \beta_{3} \rho_{i} \mu_{1} I_{\mathrm{UH}}^{2}\right)}{\left(\mu_{m}+\delta_{m}+\alpha_{m}\right) k_{3}}, \\
& I_{m}^{*}=\frac{\left(K b \varphi_{m} \alpha_{m} \beta_{2} I_{\mathrm{UH}}^{*}+b_{1} \varphi_{m} \alpha_{m} \beta_{3} \rho_{i} \mu_{1} I_{\mathrm{UH}}^{2}\right)}{k_{2} k_{3}}, \\
& M^{*}=\frac{\mu_{1} I_{\mathrm{UH}}^{*}}{\mu_{0}},
\end{aligned}
$$


where $\beta_{1}=\beta_{\mathrm{mh}}, \beta_{2}=\beta_{\mathrm{hm}}, \beta_{3}=\beta_{\mathrm{Ahm}}, k=\mu_{0}\left(\mu_{h}+\delta_{m}+\gamma_{m}+\right.$ $\left.a_{\mathrm{LI}}\right), \quad k_{1}=k \varphi_{h}\left(\mu_{h}+\gamma_{1}\right)+k \gamma_{1}\left(\gamma+\gamma_{0} u_{1}\right) I_{1}+\gamma_{1} \gamma_{m} \rho_{i} \mu_{1} I_{\mathrm{UH}}^{2}$, $k_{2}=\left(\mu_{m}+\delta_{m}\right)\left(\mu_{m}+\delta_{m}+\alpha_{m}\right)$, and $k_{3}=k\left(\mu_{m}+\delta_{m}\right)+$ $k b \beta_{2} I_{\mathrm{UH}}^{*}+b_{1} \beta_{3} \rho_{i} \mu_{1} I_{\mathrm{UH}}^{2}$. This is a positive solution of equation given by

$$
A_{1} I_{\mathrm{UH}}^{*}+A_{2} I_{\mathrm{UH}}^{*}+A_{3} I_{\mathrm{UH}}^{*}+A_{4}=0
$$

where

$$
\begin{aligned}
& A_{1}=\left\{\frac{\left(b_{1} \beta_{3} \rho_{i}^{2} \mu_{1}^{2} \mu_{0}\right) \gamma_{1} \gamma_{m}}{\varphi_{h} \alpha_{m} \beta_{2}}\left[\frac{\varphi_{h} \alpha_{m} \beta_{2}\left(\mu_{h}+\gamma_{1}\right)\left(\mu_{h}+\delta_{h}+\gamma_{m}\right)}{\gamma_{1} \gamma_{m}\left(\mu_{m}+\delta_{m}\right)\left(\mu_{h}+\delta_{h}+\gamma+\gamma_{0} u_{1}\right)}\right]-R_{0}^{2}+\left(\begin{array}{c}
k b b_{1} \alpha_{m} \varphi_{m} \beta_{1} \beta_{3} \rho_{i}^{2} \mu_{1}^{2} \mu_{0}\left(\mu_{h}+\gamma_{1}\right) \\
\left(\mu_{h}+\alpha_{h}\right)\left(\mu_{m}+\delta_{m}+\gamma_{m}\right)
\end{array}\right)\right\} \\
& A_{1}=\left\{\frac{\left(b_{1} \beta_{3} \rho_{i}^{2} \mu_{1}^{2} \mu_{0}\right) \gamma_{1} \gamma_{m}}{\varphi_{h} \alpha_{m} \beta_{2}}\left[\frac{\varphi_{h} \alpha_{m} \beta_{2}\left(\mu_{h}+\gamma_{1}\right)\left(\mu_{h}+\delta_{h}+\gamma_{m}\right)}{\gamma_{1} \gamma_{m}\left(\mu_{m}+\delta_{m}\right)\left(\mu_{h}+\delta_{h}+\gamma+\gamma_{0} u_{1}\right)}\right]-R_{0}^{2}+\left(\begin{array}{c}
k b b_{1} \alpha_{m} \varphi_{m} \beta_{1} \beta_{3} \rho_{i}^{2} \mu_{1}^{2} \mu_{0}\left(\mu_{h}+\gamma_{1}\right) \\
\left(\mu_{h}+\alpha_{h}\right)\left(\mu_{m}+\delta_{m}+\gamma_{m}\right)
\end{array}\right)\right\}, \\
& A_{3}=\left\{\frac{k^{2} \mu_{0}}{\varphi_{h}}\left[\frac{b \varphi_{h} \beta_{1}}{\gamma_{1}\left(\gamma-\gamma_{0} u_{1}\right)\left(\mu_{m}+\delta_{m}\right)}-R_{0}^{2}\right]+\frac{k\left(b_{1} \beta_{3} \rho_{i} \mu_{1} \mu_{0}\right)\left(\mu_{h}+\gamma_{1}\right)}{\left(\delta_{h}+\mu_{h}+\gamma+\gamma_{0} u_{1}\right) b \beta_{2}}\left[\frac{K b \beta_{2}}{b_{1} \beta_{3} \mu_{0}\left(\delta_{h}+\mu_{h}+\gamma+\gamma_{0} u_{1}\right)}-R_{0}^{2}\right]\right. \\
& \left.+\frac{k^{2} \mu_{0}\left(\mu_{h}+\gamma_{1}\right) a}{\varphi_{h} \alpha_{h} \mu_{h}}\left[R_{0}^{2}-\frac{\varphi_{h} \alpha_{h} \rho_{i} \mu_{1} a_{L I}}{K\left(\delta_{h}+\mu_{h}+\gamma+\gamma_{0} u_{1}\right)}\right]\right\} \\
& A_{4}=\left[k^{2} \mu_{0}\left(\mu_{h}+\gamma_{1}\right)\left(1-R_{0}^{2}\right)\right] \text {. }
\end{aligned}
$$

The systems (1)-(9) have no positive solution; when $R_{0}<1, A_{1}>0, A_{2}>0, A_{3}>0$, and $A_{4}>0$ However, when $R_{0}>1, A_{4}<0$, by using Descartes's rule of sign, there is exactly one sign-change in the equation, and there exists exactly one positive root. This implies that a unique endemic equilibrium exists. This completes the proof.

Remark 2. There are more than one endemic equilibrium existing in case, when $R_{0}^{2}>\left(\varphi_{h} \alpha_{m} \beta_{2}\left(\mu_{h}+\gamma_{1}\right)\left(\mu_{h}+\delta_{h}+\gamma_{m}\right) /\right.$ $\left.\gamma_{1} \gamma_{m}\left(\mu_{m}+\delta_{m}\right)\left(\mu_{h}+\delta_{h}+\gamma+\gamma_{0} u_{1}\right)\right)$.

\section{Results}

The general overview of the proposed model has been shown in Figure 1. The definitions and corresponding numeric values of the parameters/variables in the system of equations (1)-(9) have been summarized in Table 1. The definitions of the compartment variables are summarized in Table 2.

Figure 2 represents the 3D-plot for the proposed model. In this figure, the proposed models converge to the same point on different initial conditions. A comparison result of proposed model (incorporating awareness) and existing model (without awareness) has been shown in Figures 3-7 for human and mosquito populations. In each figure, reduction/growth of human and mosquito population in each class has been presented.

In Figures 3-5, the population of susceptible, unaware infected, and aware infected human population with and without awareness has been shown. We can easily see that, when there is no awareness, the population of susceptible humans is decaying rapidly (the red line) and there is a rapid increase in unaware infected human growth (the red line). But, when we give awareness to unaware infected humans, we experienced susceptible humans are increasing and unaware infected humans are decreasing significantly. It is due to the fact that, when we provide awareness to unaware infected humans, the contact rate of infected human and infected mosquitoes has also been reduced. The result revealed that a significant reduction in the population of unaware infected humans, for different timespans, is achieved through the proposed model. It has also been shown that the corresponding growth of unaware infected human population and infected mosquitoes increases without awareness as compared with that of the proposed model. The results also revealed that a significant reduction in the population of susceptible mosquitoes and infected mosquitoes for different timespans are achieved through the proposed model. It has also been shown that the corresponding growth of infected mosquito population is higher without awareness as compared with the proposed model.

In Figures 8 and 9, we show that, when we give awareness, it also affects the contact rate between human and mosquito population.

Figures 8 and 9 describe that, when there is no awareness, the biting rate is also high in the region. And, infected human and mosquito population increases rapidly, but when we add awareness into the system, the contact rate between aware infected humans and mosquitoes has been reduced. We show the results on the different biting rates in Figures 8 and 9 with low and high awareness rates.

\section{Discussion}

Malaria is a mosquito vector-borne disease spread in around hundred countries worldwide. The highest mortality rates are reported in tropical countries likewise sub-Saharan Africa [33]. 
TABle 2: Definition and values of variables used in the model.

\begin{tabular}{|c|c|c|c|}
\hline Variables & Corresponding definition & I. Values & Reference \\
\hline$S_{h}$ & Represents the number of individuals not yet infected with the malaria parasite, at time $t$ & 100 & [3] \\
\hline$E_{h}$ & Represents the number of individuals who are infected but not yet infectious & 20 & [3] \\
\hline$I_{\mathrm{uh}}$ & $\begin{array}{l}\text { Represents the individuals that are infected and are capable of transmitting the disease to susceptible } \\
\text { mosquitoes }\end{array}$ & 10 & [3] \\
\hline$I_{\mathrm{Ah}}$ & Represent the number of aware infected human in the region & 0 & Assumed \\
\hline$R_{h}$ & Represents the number of individuals who have temporarily recovered from the disease & 0 & [3] \\
\hline$S_{m}$ & Represents the number of mosquitoes not yet infected with the malaria parasite at time $t$ & 1000 & [3] \\
\hline$E_{m}$ & Represents the number of mosquitoes who are infected but not yet infectious & 30 & [3] \\
\hline$I_{m}$ & $\begin{array}{c}\text { Represents the mosquitoes that are infected and are capable of transmitting the disease to susceptible } \\
\text { human }\end{array}$ & 20 & [3] \\
\hline$M$ & Represents the cumulative density of awareness programs initially & 0 & Assumed \\
\hline
\end{tabular}

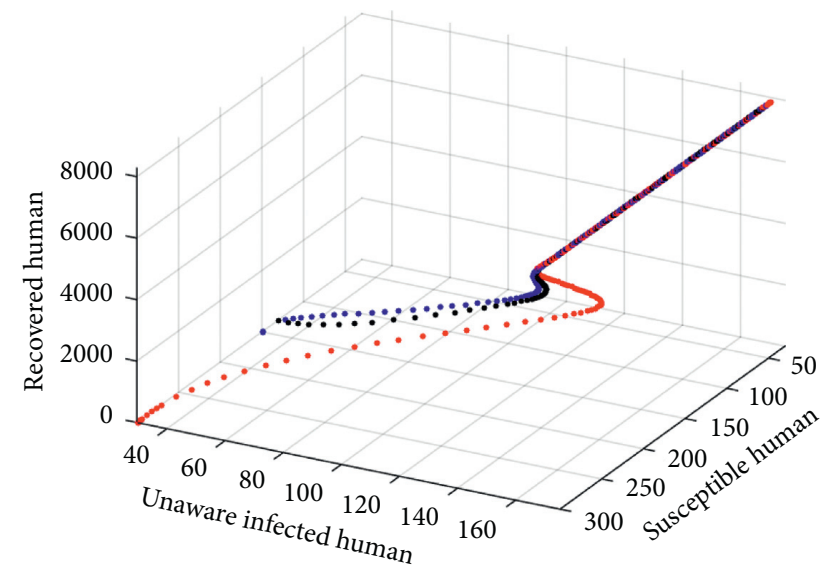

FIgURE 2: Convergence of solutions with different initial conditions.

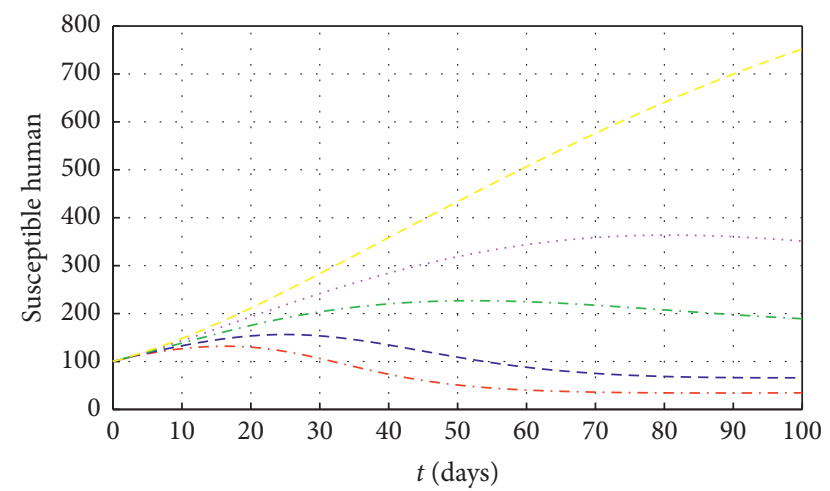

Figure 3: Population of susceptible humans with and without awareness (without awareness (red line), with low to high awareness rate (blue, green, magenta, and yellow line)).

During the past decade, several prevention measures have been used to reduce the transmission of this deadly disease. The most frequently used measures include indoor spraying and bed nets. The vaccine of this deadly disease has not been prepared till date. The measures that can reduce the spread of this dangerous disease are preventive measures and awareness. In this paper, a mathematical model for the vector-borne disease has been proposed incorporating the awareness against this disease.

Vector-borne disease models have been proposed/modified in the past. Tumwiinw and co-authors proposed a

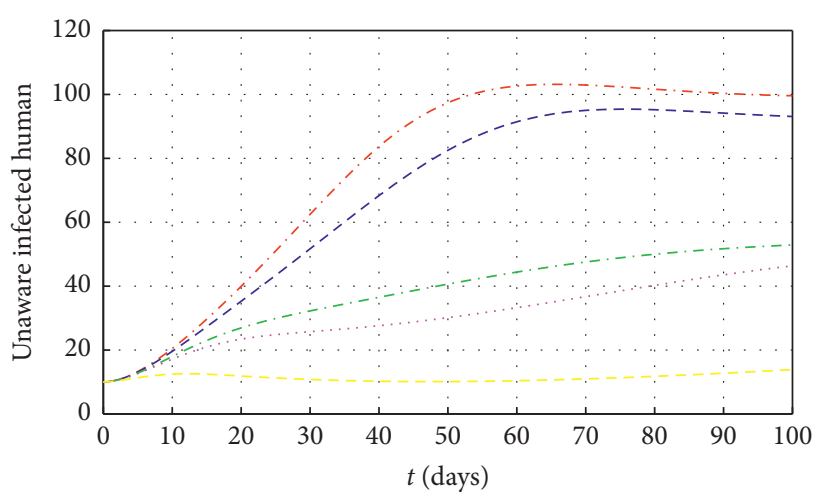

Figure 4: Population of unaware infected humans with and without awareness, (without awareness (red line); with low to high awareness rate awareness (blue, green, magenta, and yellow line)).

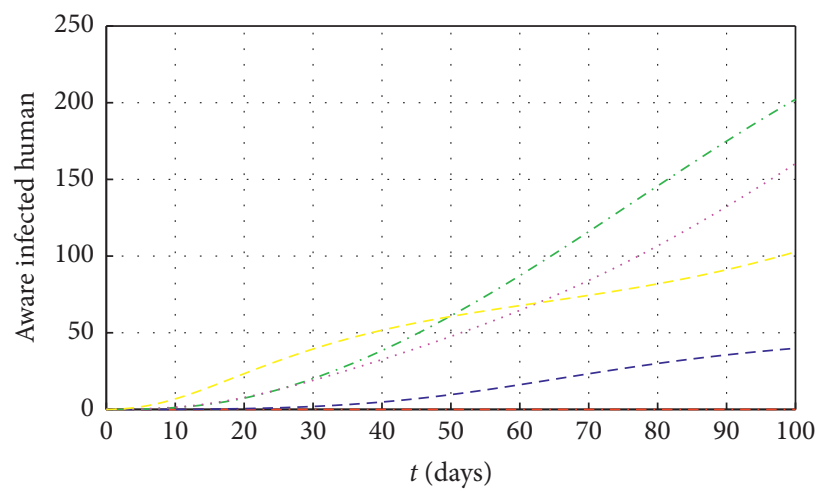

FIgURE 5: Population of aware infected humans with and without awareness (without awareness (red line); with low to high awareness rate awareness (blue, green, magenta, and yellow line)).

mathematical model that tracks the dynamics of malaria in the human host and mosquito vector [34]. They incorporated infected humans recovered from infections and immune humans after the loss of immunity against this deadly disease to rejoin the susceptible class again. Addawe and Lope have modified and analyzed the mathematical model of Tumwiinw and co-authors [17]. They divided the human population into two compartments: preschool (up to five years of age) and the rest of human population (older than five years of age). Lashari and co-authors introduced three types of control 


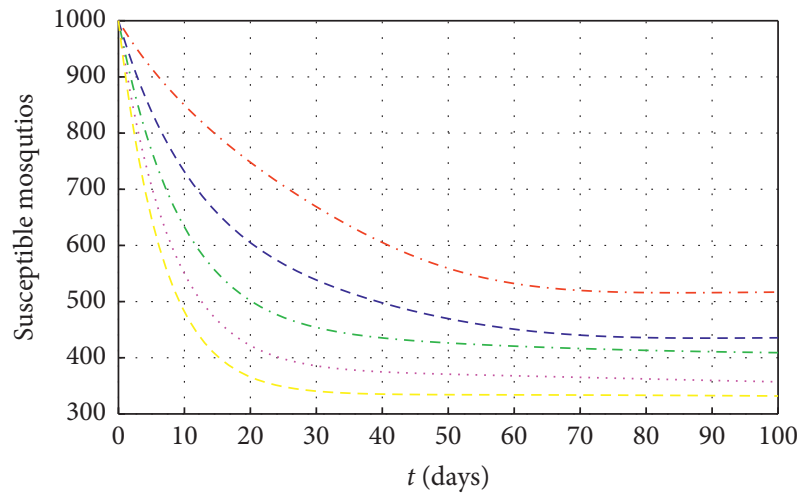

Figure 6: Population of susceptible mosquitoes with and without awareness (without awareness (red line); with low to high awareness rate awareness (blue, green, magenta, and yellow line)).

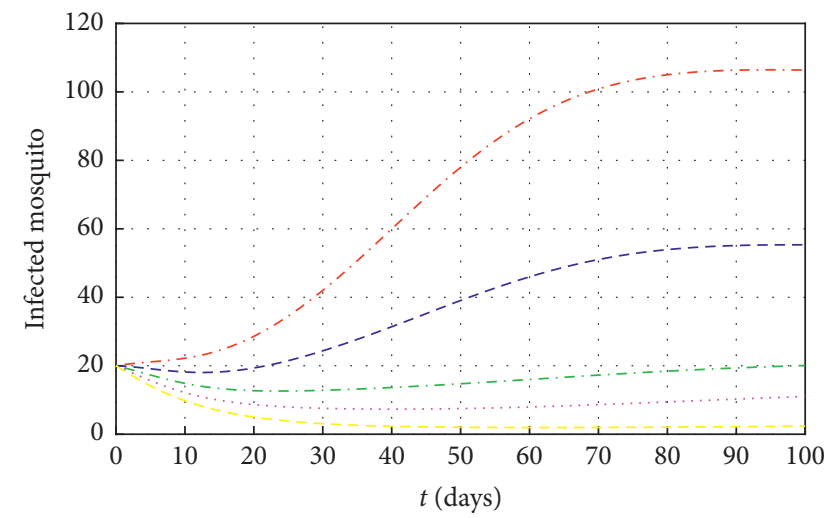

FIGURE 7: Population of infected mosquitoes with and without awareness (without awareness (red line); with low to high awareness rate awareness (blue, green, magenta, and yellow line)).

parameters, namely, personal protection, treatment, and mosquito-reduction strategies to reduce the spread of malaria disease [3]. Thus, the previous literature suggests that the reduction of the spread of this dangerous disease could be achieved through preventive measures. Therefore, a mathematical model has been developed in this study to cater the awareness strategy as a variable in the model. The awareness could be addressed to infected humans, and the aware infected humans could reduce contact rate with mosquitoes due to awareness. The proposed model has been compared with existing model. The comparative results show that a significant improvement in the reduction of vector-borne infection could be achieved with awareness.

\section{Conclusion}

Malaria is a tropical infectious disease. Scientists have not succeeded till date to develop an effective vaccine to retaliate this deadly disease. Thus, the mathematical modeling of this disease has a crucial role to understand the insights of the transmission dynamics and corresponding appropriate prevention strategies. A novel mathematical model has been proposed in this study to prevent malaria disease.

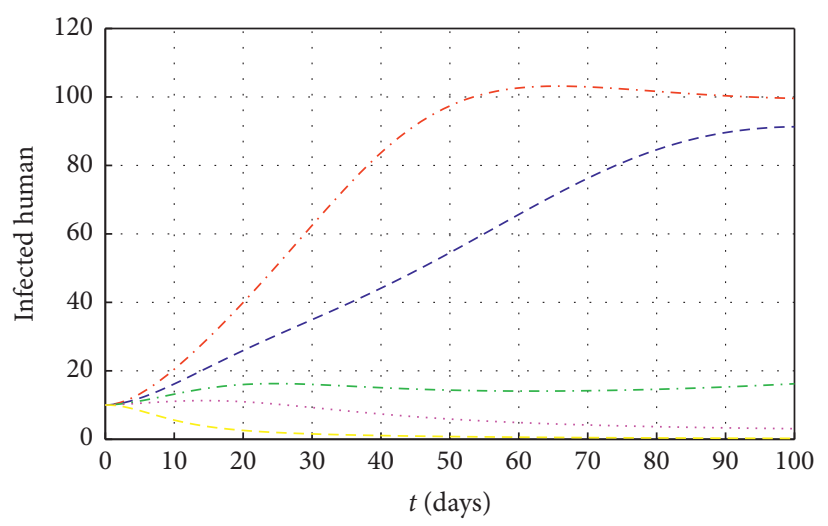

Figure 8: Population of unaware infected humans with different biting rates after awareness (without awareness (red line); with low to high awareness rate (blue, green, magenta, and yellow line)).

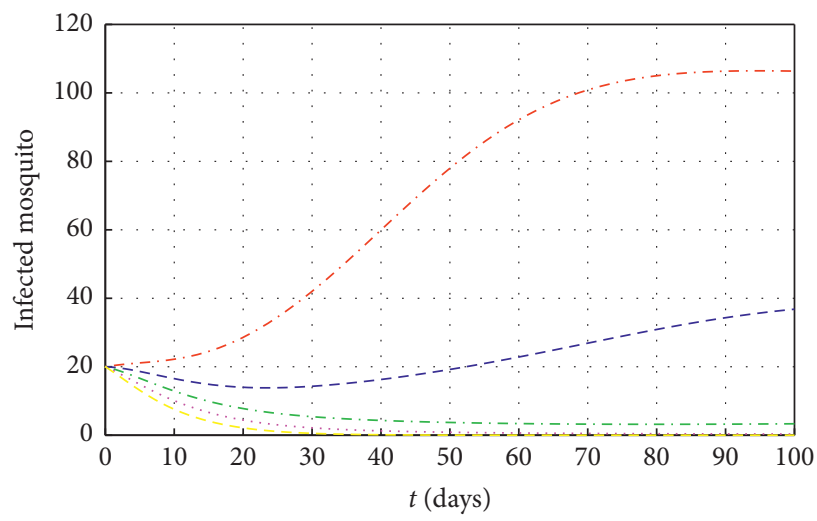

FIgURE 9: Population of infected mosquitoes with different biting rates (without awareness (red line); with low to high awareness rate (blue, green, magenta, and yellow line)).

The simulation results have significantly shown that awareness is an important factor to fight against this deadly disease. Thus, the spread of this illness could be prevented through effective awareness strategies in regions, where it has rapid spread.

\section{Data Availability}

No data has been used in this study.

\section{Conflicts of Interest}

The authors declare that they have no conflicts of interest.

\section{Acknowledgments}

This research was supported by the National Research Foundation of Korea (NRF) grants funded by the Korea government (MSIT) (NRF-2019R1A2C2007249). This research was also supported by the Basic Science Research Program through the National Research Foundation of Korea (NRF) grant funded by the Ministry of Science, ICT and Future Planning (NRF-2017R1E1A1A03070224 and NRF-2017R1A5A1015722). 


\section{References}

[1] L. Cai, N. Tuncer, and M. Martcheva, "How does within-host dynamics affect population-level dynamics? insights from an immuno-epidemiological model of malaria," Mathematical Methods in the Applied Sciences, vol. 40, no. 18, pp. 6424-6450, 2017.

[2] World Health Organization, World Malaria Report 2019, World Health Organization, Geneva, Switzerland, 2019.

[3] A. A. Lashari, S. Aly, K. Hattaf, G. Zaman, I. H. Jung, and X.-Z. Li, "Presentation of malaria epidemics using multiple optimal controls," Journal of Applied Mathematics, vol. 2012, Article ID 946504, 2012.

[4] R. Ross, The Prevention of Malaria, John Murray.[Google Scholar], London, UK, 2nd edition, 1911.

[5] M. Ozair, A. A. Lashari, I. H. Jung, Y. I. Seo, and B. N. Kim, "Stability analysis of a vector-borne disease with variable human population," Abstract and Applied Analysis, vol. 2013, Article ID 293293, 2013.

[6] R. M. Anderson and R. M. May, Infectious Diseases of Humans: Dynamics and Control, Oxford University Press, Oxford, UK, 1992.

[7] G. Macdonald, The Epidemiology and Control of Malaria, Oxford University Press, London, UK, 1957.

[8] J. L. Aron, "Mathematical modeling of immunity to malaria," Mathematical Biosciences, vol. 90, no. 1-2, pp. 385-396, 1988.

[9] J. A. N. Filipe, E. M. Riley, C. J. Drakeley, C. J. Sutherland, and A. C. Ghani, "Determination of the processes driving the acquisition of immunity to malaria using a mathematical transmission model," PLoS Computational Biology, vol. 3, no. 12, p. e255, 2007.

[10] G. Hasibeder and C. Dye, "Population dynamics of mosquitoborne disease: persistence in a completely heterogeneous environment," Theoretical Population Biology, vol. 33, no. 1, pp. 31-53, 1988.

[11] D. RodrÍguez and L. Torres-Sorando, "Models of infectious diseases in spatially heterogeneous environments," Bulletin of Mathematical Biology, vol. 63, no. 3, pp. 547-571, 2001.

[12] G. A. Ngwa and W. S. Shu, "A mathematical model for endemic malaria with variable human and mosquito populations," Mathematical and Computer Modelling, vol. 32, no. 7-8, pp. 747-764, 2000.

[13] L.-M. Cai, A. A. Lashari, I. H. Jung, K. O. Okosun, and Y. I. Seo, "Mathematical analysis of a malaria model with partial immunity to reinfection," Abstract and Applied Analysis, vol. 2013, Article ID 405258, 2013.

[14] N. Chitnis, J. M. Cushing, and J. M. Hyman, "Bifurcation analysis of a mathematical model for malaria transmission," SIAM Journal on Applied Mathematics, vol. 67, no. 1, pp. 24-45, 2006.

[15] O. Diekmann, J. A. P. Heesterbeek, and J. A. Metz, "On the definition and the computation of the basic reproduction ratio $R_{0}$ in models for infectious diseases in heterogeneous populations," Journal of Mathematical Biology, vol. 28, no. 4, pp. 365-382, 1990.

[16] M. Ozair, A. A. Lashari, I. H. Jung, and K. O. Okosun, "Stability analysis and optimal control of a vector-borne disease with nonlinear incidence," Discrete Dynamics in Nature and Society, vol. 2012, Article ID 595487, 2012.

[17] J. M. Addawe and J. E. C. Lope, "Analysis of age-structured malaria transmission model," Philippine Science Letters, vol. 5, no. 2, p. 169, 2012.

[18] A. Misra, A. Sharma, and J. Shukla, "Modeling and analysis of effects of awareness programs by media on the spread of infectious diseases," Mathematical and Computer Modelling, vol. 53, no. 5-6, pp. 1221-1228, 2011.
[19] S. Samanta, S. Rana, A. Sharma, A. K. Misra, and J. Chattopadhyay, "Effect of awareness programs by media on the epidemic outbreaks: a mathematical model," Applied Mathematics and Computation, vol. 219, no. 12, pp. 69656977, 2013.

[20] D. Greenhalgh, S. Rana, S. Samanta, T. Sardar, S. Bhattacharya, and J. Chattopadhyay, "Awareness programs control infectious disease-multiple delay induced mathematical model," Applied Mathematics and Computation, vol. 251, pp. 539-563, 2015.

[21] L. Cai, X. Li, N. Tuncer, M. Martcheva, and A. A. Lashari, "Optimal control of a malaria model with asymptomatic class and superinfection," Mathematical Biosciences, vol. 288, pp. 94-108, 2017.

[22] S. Kim, M. A. Masud, G. Cho, and I. H. Jung, "Analysis of a vector-bias effect in the spread of malaria between two different incidence areas," Journal of Theoretical Biology, vol. 419, pp. 66-76, 2017.

[23] S. Kim, J. H. Byun, A. Park, and I. H. Jung, "A mathematical model for assessing the effectiveness of controlling relapse in plasmodium vivax malaria endemic in the Republic of Korea," PLoS One, vol. 15, no. 1, Article ID e0227919, 2020.

[24] M. Ghosh, A. A. Lashari, and X.-Z. Li, "Biological control of malaria: a mathematical model," Applied Mathematics and Computation, vol. 219, no. 15, pp. 7923-7939, 2013.

[25] S. Olaniyi and O. Obabiyi, "Mathematical model for malaria transmission dynamics in human and mosquito populations with nonlinear forces of infection," International Journal of Pure and Applied Mathematics, vol. 88, no. 1, pp. 125-156, 2013.

[26] P. Van Den Driessche and J. Watmough, "Reproduction numbers and sub-threshold endemic equilibria for compartmental models of disease transmission," Mathematical Biosciences, vol. 180, no. 1-2, pp. 29-48, 2002.

[27] J. H. Jung, A. Park, and I. H. Jung, "Qualitative and sensitivity analysis of the effect of electronic cigarettes on smoking cessation," Computational and Mathematical Methods in Medicine, vol. 2018, Article ID 3738584, , 2018.

[28] K. S. Kim, M. M. Ibrahim, I. H. Jung, and S. Kim, "Mathematical analysis of the effectiveness of control strategies to prevent the autorun virus transmission propagation," Applied Mathematics and Computation, vol. 371, Article ID 124955, 2020.

[29] B. C. Kuo and F. Golnaraghi, Automatic Control Systems, Englewood Cliffs, Bergen, NJ, USA, 2003.

[30] K. Blayneh, Y. Cao, Y. Cao, and H.-D. Kwon, "Optimal control of vector-borne diseases: treatment and prevention," Discrete \& Continuous Dynamical Systems-B, vol. 11, no. 3, pp. 587-611, 2009.

[31] J. M. Tchuenche, N. Dube, C. P. Bhunu, R. J. Smith, and C. T. Bauch, "The impact of media coverage on the transmission dynamics of human influenza," BMC Public Health, vol. 11, no. S1, p. S5, 2011.

[32] C. Castillo-Chavez, Z. Feng, and W. Huang, "On the computation of $R_{0}$ and its role on global stability," Mathematical Approaches for Emerging and Reemerging Infectious Diseases: an Introduction, vol. 1, pp. 229-250, 2002.

[33] C. N. Ngonghala, S. Y. Del Valle, R. Zhao, and J. MohammedAwel, "Quantifying the impact of decay in bed-net efficacy on malaria transmission," Journal of Theoretical Biology, vol. 363, pp. 247-261, 2014.

[34] J. Tumwiine, J. Y. T. Mugisha, and L. S. Luboobi, "A mathematical model for the dynamics of malaria in a human host and mosquito vector with temporary immunity," Applied Mathematics and Computation, vol. 189, no. 2, pp. 1953-1965, 2007. 